\title{
Fluoride, Total Dissolved Solid and Electrical Conductivity in Drinking Water Supplies Analyzed in EPHI from April 2017 to December 2018
}

\author{
Tassew Arega ${ }^{1,}$,, Belaynesh Demissie ${ }^{1}$, Abel Weldetinsae ${ }^{2}$, Daniel Abera $^{2}$, Melaku Gizaw $^{2}$, \\ Tsegereda Assefa ${ }^{2}$ \\ ${ }^{1}$ Applied Chemistry, Environmental Public Health Research Team, Ethiopian Public Health Institute, Addis Ababa, Ethiopia \\ ${ }^{2}$ Environmental Science, Environmental Public Health Research Team, Ethiopian Public Health Institute, Addis Ababa, Ethiopia
}

Email address:

seidarega33@gmail.com (T. Arega),dbelaynesh87@gmail.com (B. Demissie), abelweldetinsae@gmail.com (A. Weldetinsae), daniel.aberad@gmail.com (D. Abera),melakugizaw@gmail.com (M. Gizaw), ruthtir9@yahoo.com (T. Assefa)

${ }^{*}$ Corresponding author

\section{To cite this article:}

Tassew Arega, Belaynesh Demissie, Abel Weldetinsae, Daniel Abera, Melaku Gizaw, Tsegereda Assefa. Fluoride, Total Dissolved Solid and Electrical Conductivity in Drinking Water Supplies Analyzed in EPHI from April 2017 to December 2018. International Journal of Environmental Chemistry. Vol. 3, No. 1, 2019, pp. 43-52. doi: 10.11648/j.ijec.20190301.16

Received: April 17, 2019; Accepted: June 17, 2019; Published: July 11, 2019

\begin{abstract}
This retrospective study is aim to examine the Fluoride, Total dissolved solid and Electrical conductivity in drinking water supplies of Ethiopia. The study used 345 water samples data that collected from seven regions plus two administrative cities of the country, which were tested in Environmental Public Health Chemistry Laboratory at Ethiopian Public Health Institute from April 2017 to December 2018 and from these 226, were from well water, 97 from piped and the remaining 22 were from spring water samples. The results of the water samples analysis indicate that the fluoride concentration, total dissolved solid and electrical conductivity in the water sample varied from $0.0 \mathrm{mg} / \mathrm{L}$ to $16.96 \mathrm{mg} / \mathrm{L}, 0.25$ $\mathrm{mg} / \mathrm{L}$ to $3360 \mathrm{mg} / \mathrm{Land} 2.04 \mu \mathrm{S} / \mathrm{cm}$ to $4430 \mu \mathrm{S} / \mathrm{cm}$ respectively. Generally in analyzed data, $33.6 \%(\mathrm{n}=76), 55.8 \%(\mathrm{n}=126)$ and $54.4 \%(\mathrm{n}=123)$ of the well water samples, $60.8 \%(\mathrm{n}=59), 99 \%(\mathrm{n}=96)$ and $99 \%(\mathrm{n}=96)$ of the piped water samples and $68.2 \%$ $(\mathrm{n}=15), 91 \%(\mathrm{n}=20)$ and $91 \%(\mathrm{n}=20)$ of the spring water samples are below $0.5 \mathrm{mg} / \mathrm{L}, 500 \mathrm{mg} / \mathrm{l}$ and $700 \mu \mathrm{S} / \mathrm{cm}$ of fluoride, total dissolved solid and electrical conductivity concentration respectively. on the other hand, $24 \%(\mathrm{n}=54), 9.3 \%(\mathrm{n}=21)$ and $8.4 \%(n=19)$ of the well water samples, $7.2 \%(n=7), 1 \%(n=1)$ and $1 \%(n=1)$ of the piped water samples and $4.6 \%(n=1)$, $4.6 \%(n=1)$ and $4.6 \%(n=1)$ of the spring water samples had fluoride, total dissolved solid and electrical conductivity concentration higher than WHO and national standards maximum allowable concentration (i.e. $1.5 \mathrm{mg} / \mathrm{l}, 1000 \mathrm{mg} / \mathrm{L}$ and $1500 \mu \mathrm{S} / \mathrm{cm}$ ) respectively. According to the result obtained, the water sources require a sustainable corrective action in order to alleviate the effect of fluoride, total dissolved solid and electrical conductivity in human health. Hence, the result of this retrospective study will use as base to health authorities as well as other responsible body for the management of water supply regarding fluoride, total dissolved solid and electrical conductivity.
\end{abstract}

Keywords: Total Dissolved Solid, Electrical Conductivity, Fluoride, Piped Water, Well Water, Spring Water, Drinking Water and Ethiopia

\section{Introduction}

Fluoride, conductivity (EC) and total dissolved solids (TDS) are frequently used as water quality parameters, especially in the coastal area/lowland area. The most widely recognized fluoride bearing minerals, which constitute normal hotspot for fluoride in drinking water, are fluorspar $(\mathrm{CaF} 2)$, rock phosphate, and voracity \&phosphoresces. In many researches, it is mentioned that, the fluoride contamination in drinking water is responsible for $65 \%$ of endemic flourosis in the world $[1,2]$. Total Dissolved Solids (TDS) correlates positively with conductivity and affects $\mathrm{PH}$ that means the higher the TDS, the higher the conductivity and the lower the $\mathrm{pH}$, towards acidity. The presence of dissolved 
solids in water may affect its taste [3]. Conductivity or electrical conductivity (EC) and total dissolved solids (TDS) are frequently used as water quality parameters, especially in the coastal area/lowland area. These two parameters are indicators of salinity level which make them very useful as one way in studying seawater intrusion [4-7]. The value of EC and TDS are correlated [8-10]. EC is the measure of liquid capacity to conduct an electric charge [9, 11]. Its ability depends on dissolved ion concentrations, ionic strength, and temperature of measurements [12]. The sources of material in TDS and EC can come from nature, i.e. geological condition and seawater, and from human activities, i.e. domestic and industrial waste and also agriculture [9, 13-15].

There are many standards that govern TDS and EC in water. For health reason, desirable limit for TDS is between $500 \mathrm{mg} / \mathrm{L}$ and $1,000 \mathrm{mg} / \mathrm{L}$ and for EC is no more than 1,500 $\mu \mathrm{S} / \mathrm{cm}[16]$. Other quality standards classify these parameters based on salt content or salinity level $[17,18]$. One of the water resources dissolved ions is called fluoride. This ion may be come from artificial sources such as waste water discharges from a different of industries (e.g. glassware) or enter water form mineral deposits. When inadequate, it must be added artificially [19]. Other studies show when fluoride concentration in water is $0.1 \mathrm{mg} / \mathrm{l}$, the concentration in saliva will be $0.3 \mathrm{mg} / \mathrm{l}$, whereas an increase of water fluoride concentration to $1.2 \mathrm{mg} / \mathrm{l}$ will also increase fluoride concentration in saliva to $0.9 \mathrm{mg} / \mathrm{l}$, since most system fluoride intake is from drinking water [20]. Hence, the direct relationship between water levels of fluoride and systemic absorption [21]. High exposure to Fluoride ion leads to flourosis in its dental and skeletal forms and is endemic in countries, including India, China, Mexico, Argentina, Brazil, Saudi Arabia, United States, Uganda, Tanzania, and Ethiopia. High-risk areas are mostly located in arid and semi-arid regions that are characterized by a rapid rate of chemical weathering of geological materials, in the center of East African rift, higher levels of salinity and fluoride are the most widely known. Fluoride ion problem is not only clinical, but also social problem too [22]. Ethiopia has surface and ground water resources potential, of which ground water hasalionshare. A suitability of ground water for water resource use in the central Ethiopian Rift is hampered by water quality limitations [23]. Over $40 \%$ of deep and shallow wells are contaminated with up to $36 \mathrm{mg} / 1$, sometimes $62 \mathrm{mg} / 1$ of fluoride which is significantly higher than the (WHO) guide line of $1.5 \mathrm{mg} / \mathrm{l}$. [24], that can give rise to a number of adverse effects. Natural ground water quality is mostly affected by total dissolved solids, gases and pollutants and is controlled by presence of soluble or reactive minerals in aquifers [25]. As rock chemistry and geological process like weathering of rocks, which promotes the availability of fluoride ions in the ground water [26] needs to be characterized in the study area. Existing methods for defluoridation of drinking water involve expensive and high technology or are slow, inefficient and unhygienic [22, 24]. Also, there is a gap concerning documentation of past experience, evaluating the existing performance of the spatial aquifer system chemical properties and introduction of quality improvement techniques study area.

The East African Rift Valley, which cuts through Ethiopia, is geomorphologically still an active volcanic region. The volcanic rocks, particularly in the young basalt, contain high concentrations of fluoride and fluorapatite. Large fault systems in the Valley create conditions that allow very deep percolation of infiltrating surface water. The floor of the Rift Valley that is characterized by high hydrothermal activity accelerates the solubility of fluoride. The hot climate and high fluoride waterbed of the Rift Valley, therefore, favor the development of endemic fluorosis. The water supplies in the Ethiopian Rift Valley region come mainly from boreholes with depths from 10 to 100 meters [27].

\section{Materials and Methods}

\section{Country Description}

Ethiopia found in the Horn of Africa and located between $33^{\circ} \mathrm{E}$ and $48^{\circ} \mathrm{E}$ longitudes and $3^{\circ} \mathrm{N}$ and $15^{\circ} \mathrm{N}$ of the equator. Ethiopia is country with a great geographical variation. its topography ranging from 4550 meters above sea level to 110 meters below and bordered by five countries: on the north and northeast by Eritrea, on the east by Djibouti and Somalia, on the south by Kenya, and on the west and southwest by Sudan. Ethiopia is a Federal Democratic Republic composed of nine National Regional States: namely Tigray, Afar, Amhara, Oromia, Somali, Benshangul-Gumuz, Southern Nations Nationality and People Region (SNNPR), Gambella and Harari, plus two Administrative States (Addis Ababa and Dire Dawa City Administration). The national regional states as well as the two cities Administrative councils are further divided in eight hundred woredas and around 15,000 Kebeles (5,000 Urban and 10,000 Rural) [28].

In this retrospective study 345 water samples data were used and tested in Environmental Public Health Chemistry Laboratory at Ethiopian Public Health Institute from April 2017 to December 2018. The samples data were collected from seven regions plus two administrative cities of the country and were classified based on the source type as spring, well, and piped water. The fluoride concentration in the water sample were measured using the Colorimetric SPADNS Method, under acidic condition fluorides (HF) react with zirconium SPADNS solution and the lake (color of SPADNS reagent) gets bleaching due to formation of $\mathrm{ZrF} 6$. Since bleaching is a function of fluoride ions, it is directly proportional to the concentration of fluoride. It obeys Beers law in a reverse manner and calculated as $\mathrm{mg} \mathrm{F}$ - /L present in the sample using standard curve, according to Standard Method of Water and Wastewater analysis [29]. Electrical conductivity and Total dissolved solid were determined by a portable Electrical conductivity and Total dissolved solid meter model (Model Sx713). An Electrical conductivity and Total dissolved solid were calibrated before measurement with an Electrical conductivity reference solution at $25^{\circ} \mathrm{C}$ according to the manufacturer's calibration procedure. 


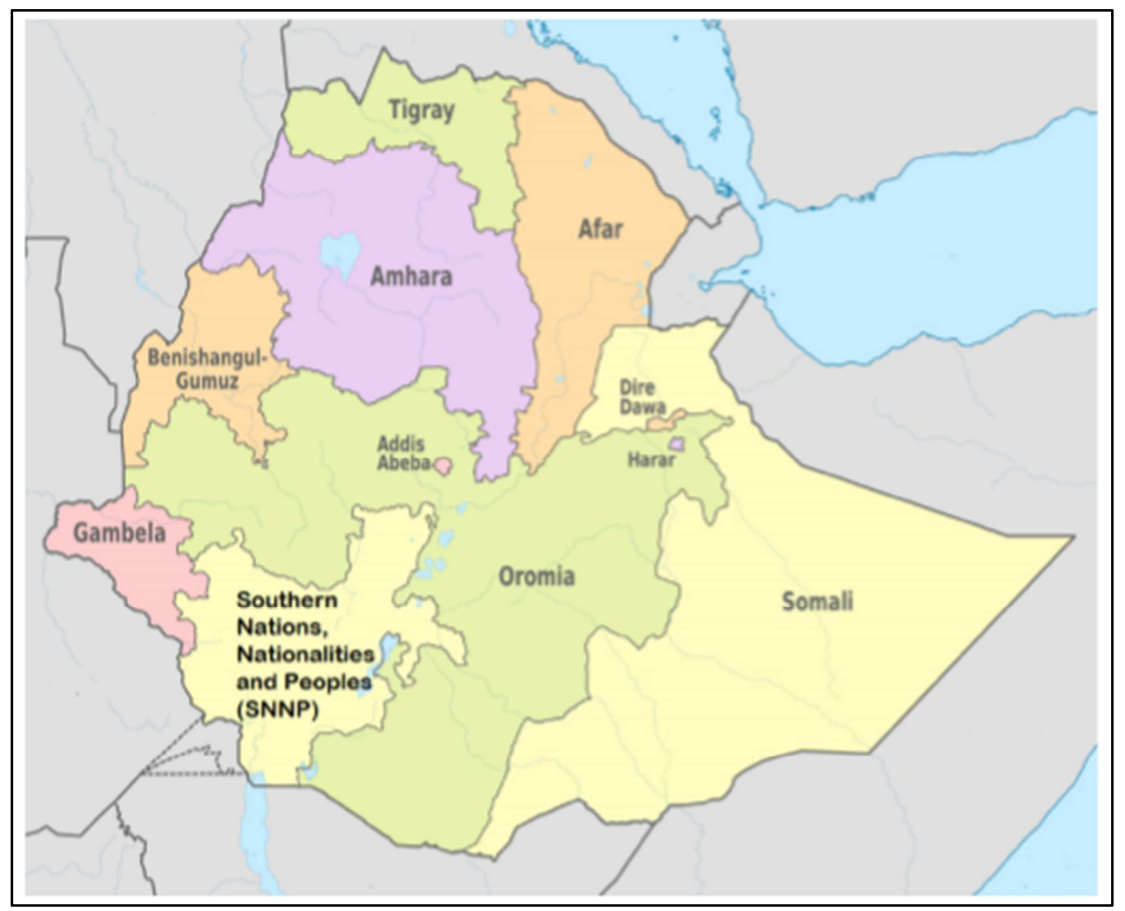

Figure 1. Map of Ethiopian regional and administrative city.

\section{Result and Discussion}

Fluoride concentration in the water sample that collects from different regions of the country varied from $0.0 \mathrm{mg} / \mathrm{L}$ to
$16.96 \mathrm{mg} / \mathrm{L}$.

The concentration of the sample classified into five groups based on WHO guidelines for fluoride in drinking water [30].

Table 1. Fluoride concentration in well water collected from different regions of Ethiopia.

\begin{tabular}{|c|c|c|c|c|c|c|c|c|c|c|c|c|c|}
\hline \multirow{3}{*}{ Region } & \multirow{3}{*}{ Total $* \mathbf{N}$ o } & \multicolumn{12}{|c|}{ Concentration of fluoride (mg/l) } \\
\hline & & \multicolumn{2}{|l|}{$<0.5$} & \multicolumn{2}{|c|}{$0.5-1.5$} & \multicolumn{2}{|l|}{$1.5-4$} & \multicolumn{2}{|l|}{ 4-10 } & \multicolumn{2}{|l|}{$>10$} & \multirow{2}{*}{ Mean value } & \multirow{2}{*}{ Max.Value } \\
\hline & & $* \mathbf{N}_{0}$ & $(\%)$ & $* \mathbf{N}_{0}$ & $(\%)$ & $* \mathbf{N}_{\mathbf{0}}$ & $(\%)$ & $* \mathbf{N}_{\mathbf{0}}$ & $(\%)$ & $* \mathbf{N}_{0}$ & $(\%)$ & & \\
\hline Addis Ababa & 42 & 25 & 59.5 & 16 & 38.1 & 1 & 2.3 & - & - & - & - & 0.49 & 2.81 \\
\hline Oromia & 126 & 38 & 30.2 & 42 & 33.3 & 9 & 7.1 & 33 & 26.2 & 4 & 3.2 & 2.47 & 16.96 \\
\hline Tigray & 6 & 4 & 66.7 & - & - & 1 & 16.7 & 1 & & - & - & 1.28 & 4.18 \\
\hline Amhara & 7 & 4 & 57.1 & 3 & 42.9 & - & - & - & - & - & - & 0.42 & 1.12 \\
\hline Somalia & 16 & 1 & 6.25 & 13 & 81.3 & 2 & 12.5 & - & - & - & - & 1.36 & 5.1 \\
\hline Afar & 20 & 1 & 5 & 17 & 85 & 2 & 10 & - & - & - & - & 0.94 & 2.54 \\
\hline SNNP & 5 & - & - & 4 & 80 & 1 & 20 & - & - & - & - & 0.92 & 1.72 \\
\hline Harari & 2 & 1 & 50 & 1 & 50 & - & - & - & - & - & - & 0.5 & 0.63 \\
\hline DireDawa & 2 & 2 & 100 & - & - & - & - & - & - & - & - & 0.36 & 0.4 \\
\hline
\end{tabular}

Where *No stands for Number of Sample SNNPR- Southern Nation Nationality People Region

Table 2. Fluoride concentration in piped water collected from different regions of Ethiopia.

\begin{tabular}{|c|c|c|c|c|c|c|c|c|c|c|c|c|c|}
\hline \multirow{3}{*}{ Region } & \multirow{3}{*}{ Total $* \mathbf{N} 0$} & \multicolumn{12}{|c|}{ Concentration of fluoride (mg/l) } \\
\hline & & \multicolumn{2}{|l|}{$<0.5$} & \multicolumn{2}{|c|}{$0.5-1.5$} & \multicolumn{2}{|l|}{$1.5-4$} & \multicolumn{2}{|l|}{ 4-10 } & \multicolumn{2}{|l|}{$>10$} & \multirow{2}{*}{ Mean value } & \multirow{2}{*}{ Max. Value } \\
\hline & & $* \mathbf{N}$ o & $(\%)$ & $* \mathbf{N} \mathbf{0}$ & $(\%)$ & $* \mathbf{N}$ o & $(\%)$ & $* \mathbf{N}$ o & $(\%)$ & $* \mathbf{N} \mathbf{o}$ & $(\%)$ & & \\
\hline Oromia & 19 & 8 & 42.1 & 6 & 31.6 & 4 & 21.1 & 1 & 5.2 & - & - & 1.4 & 7.75 \\
\hline Tigray & - & - & - & - & - & - & - & - & - & - & - & - & - \\
\hline Amhara & 1 & 1 & 100 & - & - & - & - & - & - & - & - & 0.34 & 0.34 \\
\hline Afar & - & - & - & - & - & - & - & - & - & - & - & - & - \\
\hline SNNP & - & - & - & - & - & - & - & - & - & - & - & - & - \\
\hline Harari & - & - & - & - & - & - & - & - & - & - & - & - & - \\
\hline Dire Dawa & - & - & - & - & - & - & - & - & - & - & - & - & - \\
\hline
\end{tabular}


Table 3. Fluoride concentration in spring water collected from different regions of Ethiopia.

\begin{tabular}{|c|c|c|c|c|c|c|c|c|c|c|c|c|c|}
\hline \multirow{3}{*}{ Region } & \multirow{3}{*}{ Total $* \mathbf{N} 0$} & \multicolumn{12}{|c|}{ Concentration of fluoride (mg/l) } \\
\hline & & \multicolumn{2}{|l|}{$<0.5$} & \multicolumn{2}{|c|}{$0.5-1.5$} & \multicolumn{2}{|l|}{ 1.5-4 } & \multicolumn{2}{|l|}{ 4-10 } & \multicolumn{2}{|l|}{$>10$} & \multirow{2}{*}{ Mean value } & \multirow{2}{*}{ Max. Value } \\
\hline & & $* \mathbf{N o}_{0}$ & $(\%)$ & $* \mathbf{N}_{0}$ & $(\%)$ & *N o & $(\%)$ & $* \mathbf{N}_{0}$ & $(\%)$ & $* \mathbf{N o}_{0}$ & $(\%)$ & & \\
\hline Addis Ababa & - & - & - & - & - & - & - & - & - & - & - & - & - \\
\hline Oromia & 11 & 10 & 90.9 & 1 & 9.1 & - & - & - & - & - & - & 0.23 & 0.58 \\
\hline Tigray & 2 & 2 & 100 & - & - & - & - & - & - & - & - & 0.075 & 0.08 \\
\hline Amhara & 4 & 2 & 50 & 2 & 50 & - & - & - & - & - & - & 0.66 & 1.09 \\
\hline Somalia & - & - & - & - & - & - & - & - & - & - & - & - & - \\
\hline Afar & - & - & - & - & - & - & - & - & - & - & - & - & - \\
\hline SNNP & 5 & 1 & 20 & 3 & 60 & - & - & - & - & 1 & 20 & 2.62 & 10.12 \\
\hline Harari & - & - & - & - & - & - & - & - & - & - & - & - & - \\
\hline Dire Dawa & - & - & - & - & - & - & - & - & - & - & - & - & - \\
\hline
\end{tabular}

Where *No stands for Number of Sample SNNPR- Southern Nation Nationality People Region

Table 4. Fluoride concentration in well, piped and spring water collected from different regions of Ethiopia that exceeded the WHO guideline (1.5 mg/L).

\begin{tabular}{|c|c|c|c|c|c|c|c|c|c|}
\hline \multirow{2}{*}{ Region } & \multirow{2}{*}{ Well Total *No } & \multicolumn{2}{|l|}{$>1.5 \mathrm{mg} / \mathrm{L}$} & \multirow{2}{*}{ Piped Total *No } & \multicolumn{2}{|l|}{$>1.5 \mathrm{mg} / \mathrm{L}$} & \multirow{2}{*}{ Spring Total *No } & \multicolumn{2}{|l|}{$>1.5 \mathrm{mg} / \mathrm{L}$} \\
\hline & & Well *No & $(\%)$ & & Piped *No & $(\%)$ & & Spring *No & $(\%)$ \\
\hline Addis Ababa & 42 & 1 & 2.4 & 77 & 2 & 2.6 & - & - & - \\
\hline Oromia & 126 & 46 & 36.5 & 19 & 5 & 26.3 & 11 & - & - \\
\hline Tigray & 6 & 2 & 33.3 & - & - & - & 2 & - & - \\
\hline Amhara & 7 & - & - & 1 & - & - & 4 & - & - \\
\hline Somalia & 16 & 2 & 12.5 & - & - & - & - & - & - \\
\hline Afar & 20 & 2 & 10 & - & - & - & - & - & - \\
\hline Harari & 2 & - & - & - & - & - & - & - & - \\
\hline Dire Dawa & 2 & - & - & - & - & - & - & - & - \\
\hline
\end{tabular}

Where *No stands for Number of Sample SNNPR- Southern Nation Nationality People Region

Table 5. Fluoride concentrations that have $0.00 \mathrm{mg} / \mathrm{L}$ in their water source of the region of Ethiopia.

\begin{tabular}{llll}
\hline Region & Zone, wereda or another village & Source of Sample & Fluoride Concentration (mg/L) \\
\hline \multirow{2}{*}{ Addis Ababa } & Gulele sub city (woreda 9), Ethiopia pharmaceutical factory & Well \\
& Awash Wine Company, Gulele sub city (woreda 9) & piped & Well \\
Oromia & Dukem & O.0 & \\
\hline
\end{tabular}

Table 6. Fluoride concentrations that have exceeded more from WHO guideline in their drinking water source.

\begin{tabular}{|c|c|c|c|}
\hline Region & Zone, wereda or another village & Source of Sample & Fluoride Concentration $(\mathrm{mg} / \mathrm{L})$ \\
\hline \multirow{3}{*}{ Add is Ababa } & MCM Company & Well & 2.81 \\
\hline & \multirow{2}{*}{ Gulele Sub city (woreda 9) } & \multirow{2}{*}{ Piped } & 3.99 \\
\hline & & & 4.44 \\
\hline \multirow{12}{*}{ Oromia } & \multirow[t]{2}{*}{ Lume (Qoqa) } & \multirow[t]{2}{*}{ Well } & 16.96 \\
\hline & & & 8.25 \\
\hline & \multirow[t]{2}{*}{ Merti } & \multirow[t]{2}{*}{ Well } & 9.03 \\
\hline & & & 8.64 \\
\hline & Adama (Wonji) & Well & 7.67 \\
\hline & Zeway & Well & 10.12 \\
\hline & \multirow[t]{2}{*}{ Adami tulu } & \multirow[t]{2}{*}{ Well } & 10.58 \\
\hline & & & 9.05 \\
\hline & \multirow{3}{*}{ Zeway } & \multirow{3}{*}{ Well } & 9.84 \\
\hline & & & 10.62 \\
\hline & & & 9.72 \\
\hline & East shoa (Batu) & Piped & 7.75 \\
\hline \multirow{2}{*}{ Tigray } & \multirow{2}{*}{ North west (Asegede Tsebela) } & \multirow{2}{*}{ Well } & 2.73 \\
\hline & & & 4.18 \\
\hline \multirow{2}{*}{ Somalia } & D01loo Galadi & Well & 4.21 \\
\hline & Dolloo Warder & Well & 5.1 \\
\hline Afar & Dulesa & Well & 2.54 \\
\hline SNNP & Gurage (Agena) & Spring & 10.12 \\
\hline
\end{tabular}

Total dissolved solid concentration in the water sample that collects from different regions of the country varied from 0.25 $\mathrm{mg} / \mathrm{L}$ to $3360 \mathrm{mg} / \mathrm{L}$. The concentration of the sample classified into five groups based on WHO guidelines for total dissolved solid in drinking water $[31,16]$. 
Table 7. Total dissolved solid concentration in well water collected from different regions of Ethiopia.

\begin{tabular}{|c|c|c|c|c|c|c|c|c|c|c|c|c|c|}
\hline \multirow{3}{*}{ Region } & \multirow{3}{*}{ Total *N o } & \multicolumn{12}{|c|}{ Concentration of total dissolved solid (mg/l) } \\
\hline & & \multicolumn{2}{|c|}{$<\mathbf{1 0 0}$} & \multicolumn{2}{|c|}{$100-500$} & \multicolumn{2}{|c|}{$500-1000$} & \multicolumn{2}{|c|}{$1000-1500$} & \multicolumn{2}{|c|}{$>1500$} & \multirow{2}{*}{ Mean value } & \multirow{2}{*}{ Max. Value } \\
\hline & & *N o & $(\%)$ & *N o & $(\%)$ & *N o & $(\%)$ & *No & $(\%)$ & $* \mathbf{N o}$ & $(\%)$ & & \\
\hline Addis Ababa & 42 & 3 & 7.2 & 36 & 85.6 & 3 & 7.2 & - & - & - & - & 301 & 579 \\
\hline Oromia & 126 & 9 & 7.1 & 61 & 48.4 & 54 & 42.9 & 2 & 1.6 & - & - & 484.7 & 1190 \\
\hline Tigray & 6 & - & - & 2 & 33.3 & 4 & 66.7 & - & - & - & - & 515.2 & 772 \\
\hline Amhara & 7 & 2 & 28.6 & 5 & 71.4 & - & - & - & - & - & - & 208.3 & 433 \\
\hline Somalia & 16 & - & - & - & - & 6 & 37.5 & 5 & 31.2 & 5 & 31.2 & 1246.3 & 2280 \\
\hline Afar & 20 & - & - & 3 & 15 & 8 & 40 & 4 & 20 & 5 & 25 & 1196.1 & 3360 \\
\hline SNNP & 5 & - & - & 5 & 100 & - & - & - & - & - & - & 195.8 & 257 \\
\hline Harari & 2 & - & - & - & - & 2 & 100 & - & - & - & - & 747 & 931 \\
\hline Dire Dawa & 2 & - & - & - & - & 2 & 100 & - & - & - & - & 709 & 758 \\
\hline
\end{tabular}

Table 8. Total dissolved solid concentration in piped water collected from different regions of Ethiopia.

\begin{tabular}{|c|c|c|c|c|c|c|c|c|c|c|c|c|c|}
\hline \multirow{3}{*}{ Region } & \multirow{3}{*}{ Total *N o } & \multicolumn{12}{|c|}{ Concentration of total dissolved solid (mg/l) } \\
\hline & & \multicolumn{2}{|l|}{$<100$} & \multicolumn{2}{|c|}{$100-500$} & \multicolumn{2}{|c|}{$500-1000$} & \multicolumn{2}{|c|}{$1000-1500$} & \multicolumn{2}{|c|}{$>1500$} & \multirow[b]{2}{*}{ Mean value } & \multirow[b]{2}{*}{ Max. Value } \\
\hline & & $* \mathbf{N o}$ & $(\%)$ & $* \mathbf{N O}_{0}$ & $(\%)$ & $* \mathbf{N o}$ & $(\%)$ & $* \mathbf{N} 0$ & $(\%)$ & $* \mathbf{N} 0$ & $(\%)$ & & \\
\hline Addis Ababa & 77 & 23 & 29.9 & 54 & 70.1 & - & - & - & - & - & - & 201.1 & 448 \\
\hline Oromia & 19 & 4 & 21.1 & 14 & 73.7 & - & - & 1 & 5.2 & - & - & 305.3 & 1170 \\
\hline Tigray & - & - & - & - & - & - & - & - & - & - & - & - & - \\
\hline Amhara & 1 & - & - & 1 & 100 & - & - & - & - & - & - & 165 & 165 \\
\hline Somalia & - & - & - & - & - & - & - & - & - & - & - & - & - \\
\hline Afar & - & - & - & - & - & - & - & - & - & - & - & - & - \\
\hline SNNP & - & - & - & - & - & - & - & - & - & - & - & - & - \\
\hline Harari & - & - & - & - & - & - & - & - & - & - & - & - & - \\
\hline DireDawa & - & - & - & - & - & - & - & - & - & - & - & - & - \\
\hline
\end{tabular}

Where *No stands for Number of Sample SNNPR- Southern Nation Nationality People Region

Table 9. Total dissolved solid concentration in spring water collected from different regions of Ethiopia.

\begin{tabular}{|c|c|c|c|c|c|c|c|c|c|c|c|c|c|}
\hline \multirow{3}{*}{ Region } & \multirow{3}{*}{ Total $* \mathbf{N o}$} & \multicolumn{12}{|c|}{ Concentration of total dissolved solid (mg/l) } \\
\hline & & \multicolumn{2}{|c|}{$<100$} & \multicolumn{2}{|c|}{$100-500$} & \multicolumn{2}{|c|}{$500-1000$} & \multicolumn{2}{|c|}{$1000-1500$} & \multicolumn{2}{|c|}{$>1500$} & \multirow[t]{2}{*}{ Mean value } & \multirow[t]{2}{*}{ Max. Value } \\
\hline & & *No & $(\%)$ & $* \mathbf{N o}_{0}$ & (\%) & $* \mathbf{N o}$ & $(\%)$ & $* \mathbf{N o}_{0}$ & (\%) & $* \mathbf{N o}$ & $(\%)$ & & \\
\hline Addis Ababa & - & - & - & - & - & - & - & - & - & - & - & - & - \\
\hline Oromia & 11 & 8 & 72.7 & 3 & 27.3 & - & - & - & - & - & - & 81.7 & 180 \\
\hline Tigray & 2 & - & - & 2 & 100 & - & - & - & - & - & - & 207 & 226 \\
\hline Amhara & 4 & - & - & 3 & 75 & - & - & - & - & 1 & 25 & 982.5 & 3280 \\
\hline Somalia & - & - & - & - & - & - & - & - & - & - & - & - & - \\
\hline Afar & - & - & - & - & - & - & - & - & - & - & - & - & - \\
\hline SNNP & 5 & 3 & 60 & 1 & 20 & 1 & 20 & - & - & - & - & 238.5 & 773 \\
\hline Harari & - & - & - & - & - & - & - & - & - & - & - & - & - \\
\hline DireDawa & - & - & - & - & - & - & - & - & - & - & - & - & - \\
\hline
\end{tabular}

Table 10. Total dissolved solid concentration in well, piped and spring water collected from different regions of Ethiopia that exceeded the WHO guideline $(1000 \mathrm{mg} / \mathrm{L})$.

\begin{tabular}{|c|c|c|c|c|c|c|c|c|c|}
\hline \multirow{2}{*}{ Region } & \multirow{2}{*}{ Well Total *No } & \multicolumn{2}{|c|}{$>1000 \mathrm{mg} / \mathrm{L}$} & \multirow{2}{*}{ Piped Total *No } & \multicolumn{2}{|l|}{$>1000 \mathrm{mg} / \mathrm{L}$} & \multirow{2}{*}{ Spring Total *No } & \multicolumn{2}{|l|}{$>1000 \mathrm{mg} / \mathrm{L}$} \\
\hline & & Well *No & $(\%)$ & & Piped *No & $(\%)$ & & Spring *No & $(\%)$ \\
\hline Addis Ababa & 42 & - & - & 77 & - & - & - & - & - \\
\hline Oromia & 126 & 2 & 1.6 & 19 & 1 & 5.3 & 11 & - & - \\
\hline Tigray & 6 & - & - & - & - & - & 2 & - & - \\
\hline Amhara & 7 & - & - & 1 & - & - & 4 & 1 & 25 \\
\hline Somalia & 16 & 10 & 62.5 & - & - & - & - & - & - \\
\hline Afar & 20 & 9 & 45 & - & - & - & - & - & - \\
\hline SNNP & 5 & - & - & - & - & - & 5 & - & - \\
\hline Harari & 2 & - & - & - & - & - & - & - & - \\
\hline Dire Dawa & 2 & - & - & - & - & - & - & - & - \\
\hline
\end{tabular}

Where *No stands for Number of Sample SNNPR- Southern Nation Nationality People Region

Electrical conductivity in the water sample that collects from different regions of the country varied from $2.04 \mu \mathrm{S} / \mathrm{cm}$ to $4430 \mu \mathrm{S} / \mathrm{cm}$. The concentration of the sample classified into five groups based on $[18,16]$ guidelines for Electrical conductivity in drinking water. 
Table 11. Electrical conductivity in well water collected from different regions of Ethiopia.

\begin{tabular}{|c|c|c|c|c|c|c|c|c|c|c|c|c|c|}
\hline \multirow{3}{*}{ Region } & \multirow{3}{*}{ Total *N o } & \multicolumn{12}{|c|}{ Concentration of electrical conductivity $(\mu \mathrm{S} / \mathrm{cm})$} \\
\hline & & \multicolumn{2}{|l|}{$<700$} & \multicolumn{2}{|c|}{$700-1500$} & \multicolumn{2}{|c|}{$1500-10000$} & \multicolumn{2}{|c|}{$10000-45000$} & \multicolumn{2}{|c|}{$>45000$} & \multirow{2}{*}{ Mean value } & \multirow{2}{*}{ Max. Value } \\
\hline & & $* \mathrm{~N} \mathrm{o}^{2}$ & $(\%)$ & $* \mathbf{N o}$ & $(\%)$ & $* \mathbf{N o}_{0}$ & $(\%)$ & $* \mathbf{N o}$ & $(\%)$ & *No & $(\%)$ & & \\
\hline Addis Ababa & 42 & 39 & 92.8 & 3 & 7.2 & - & - & - & - & - & - & 444.5 & 843 \\
\hline Oromia & 126 & 69 & 54.8 & 56 & 44.4 & 1 & 0.8 & - & - & - & - & 703.5 & 1666 \\
\hline Tigray & 6 & 2 & 33.3 & 4 & 66.7 & - & - & - & - & - & - & 755.3 & 1107 \\
\hline Amhara & 7 & 7 & 100 & - & - & - & - & - & - & - & - & 308.8 & 636 \\
\hline Somalia & 16 & - & - & 6 & 37.5 & 10 & 62.5 & - & - & - & - & 1723.6 & 3030 \\
\hline Afar & 20 & 1 & 5 & 11 & 55 & 8 & 40 & - & - & - & - & 1647.9 & 4430 \\
\hline SNNP & 5 & 5 & 100 & - & - & - & - & - & - & - & - & 291.8 & 383 \\
\hline Harari & 2 & - & - & 2 & 100 & - & - & - & - & - & - & 1072 & 1325 \\
\hline DireDawa & 2 & - & - & 2 & 100 & - & - & - & - & - & - & 1022 & 1090 \\
\hline
\end{tabular}

Table 12. Electrical conductivity in piped water collected from different regions of Ethiopia.

\begin{tabular}{|c|c|c|c|c|c|c|c|c|c|c|c|c|c|}
\hline \multirow{3}{*}{ Region } & \multirow{3}{*}{ Total $* \mathbf{N}$ o } & \multicolumn{12}{|c|}{ Concentration of electrical conductivity $(\mu \mathrm{S} / \mathrm{cm})$} \\
\hline & & \multicolumn{2}{|c|}{$<700$} & \multicolumn{2}{|c|}{$700-1500$} & \multicolumn{2}{|c|}{$1500-10000$} & \multicolumn{2}{|c|}{$10000-45000$} & \multicolumn{2}{|c|}{$>45000$} & \multirow{2}{*}{ Mean value } & \multirow{2}{*}{ Max. Value } \\
\hline & & *N o & $(\%)$ & *No & $(\%)$ & $* \mathbf{N} \mathbf{0}$ & $(\%)$ & $* \mathbf{N}_{0}$ & $(\%)$ & *No & $(\%)$ & & \\
\hline Addis Ababa & 77 & 77 & 100 & - & - & - & - & - & - & - & - & 301 & 658 \\
\hline Oromia & 19 & 18 & 94.7 & - & - & 1 & 5.3 & - & - & - & - & 447.9 & 1638 \\
\hline Tigray & - & - & - & - & - & - & - & - & - & - & - & - & - \\
\hline Amhara & 1 & 1 & 100 & - & - & - & - & - & - & - & - & 247 & 247 \\
\hline Somalia & - & - & - & - & - & - & - & - & - & - & - & - & - \\
\hline Afar & - & - & - & - & - & - & - & - & - & - & - & - & - \\
\hline SNNP & - & - & - & - & - & - & - & - & - & - & - & - & - \\
\hline Harari & - & - & - & - & - & - & - & - & - & - & - & - & - \\
\hline DireDawa & - & - & - & - & - & - & - & - & - & - & - & - & - \\
\hline
\end{tabular}

Table 13. Electrical conductivity in spring water collected from different regions of Ethiopia.

\begin{tabular}{|c|c|c|c|c|c|c|c|c|c|c|c|c|c|}
\hline \multirow{3}{*}{ Region } & \multirow{3}{*}{ Total *N o } & \multicolumn{12}{|c|}{ Concentration of electrical conductivity $(\mu \mathrm{S} / \mathrm{cm})$} \\
\hline & & \multicolumn{2}{|c|}{$<700$} & \multicolumn{2}{|c|}{$700-1500$} & \multicolumn{2}{|c|}{$1500-10000$} & \multicolumn{2}{|c|}{$10000-45000$} & \multicolumn{2}{|c|}{$>45000$} & \multirow{2}{*}{ Mean value } & \multirow{2}{*}{ Max. Value } \\
\hline & & $* \mathbf{N o}$ & $(\%)$ & $* \mathbf{N o}$ & $(\%)$ & *N o & $(\%)$ & $* \mathbf{N} \mathbf{0}$ & $(\%)$ & $* \mathbf{N o}$ & $(\%)$ & & \\
\hline Addis Ababa & - & - & - & - & - & - & - & - & - & - & - & - & - \\
\hline Oromia & 11 & 11 & 100 & - & - & - & - & - & - & - & - & 124.1 & 270 \\
\hline Tigray & 2 & 2 & 100 & - & - & - & - & - & - & - & - & 309.5 & 337 \\
\hline Amhara & 4 & 3 & 75 & - & - & 1 & 25 & - & - & - & - & 1316 & 4300 \\
\hline Somalia & - & - & - & - & - & - & - & - & - & - & - & - & - \\
\hline Afar & - & - & - & - & - & - & - & - & - & - & - & - & - \\
\hline SNNP & 5 & 4 & 80 & 1 & 20 & - & - & - & - & - & - & 348.7 & 1113 \\
\hline Harari & - & - & - & - & - & - & - & - & - & - & - & - & - \\
\hline DireDawa & - & - & - & - & - & - & - & - & - & - & - & - & - \\
\hline
\end{tabular}

Table 14. Electrical conductivity in well, piped and spring water collected from different regions of Ethiopia that exceeded the WHO guideline (1500 $\mu$ S/cm).

\begin{tabular}{|c|c|c|c|c|c|c|c|c|c|}
\hline \multirow{2}{*}{ Region } & \multirow{2}{*}{ Well Total *No } & \multicolumn{2}{|c|}{$>1500 \mu \mathrm{S} / \mathrm{cm}$} & \multirow{2}{*}{ Piped Total *No } & \multicolumn{2}{|c|}{$>1500 \mu \mathrm{S} / \mathrm{cm}$} & \multirow{2}{*}{ Spring Total *No } & \multicolumn{2}{|c|}{$>1500 \mu \mathrm{S} / \mathrm{cm}$} \\
\hline & & Well *No & (\%) & & Piped *No & $(\%)$ & & Spring *No & $(\%)$ \\
\hline Addis Ababa & 42 & - & - & 77 & - & - & - & - & - \\
\hline Oromia & 126 & 1 & 0.8 & 19 & 1 & 5.3 & 11 & - & - \\
\hline Tigray & 6 & - & - & - & - & - & 2 & - & - \\
\hline Amhara & 7 & - & - & 1 & - & - & 4 & 1 & 25 \\
\hline Somalia & 16 & 10 & 62.5 & - & - & - & - & - & - \\
\hline Afar & 20 & 8 & 40 & - & - & - & - & - & - \\
\hline SNNP & 5 & - & - & - & - & - & 5 & - & - \\
\hline Harari & 2 & - & - & - & - & - & - & - & - \\
\hline Dire Dawa & 2 & - & - & - & - & - & - & - & - \\
\hline
\end{tabular}

Where *No stands for Number of Sample SNNPR- Southern Nation Nationality People Region

Table 15. Electrical conductivity and Total dissolved solid Concentration that have exceeded more from WHO guideline in their drinking water source.

\begin{tabular}{lllll}
\hline Region & Zone, wereda or another village & Source of Sample & Total dissolved solid Concentration $(\mathbf{m g} / \mathbf{L})$ & Electrical conductivity $(\boldsymbol{\mu S} / \mathbf{c m})$ \\
\hline \multirow{2}{*}{ Oromia } & Sululta & Well & 1190 & 1666 \\
& East Shoa (Batu) & Piped & 1170 & 1638 \\
\multirow{2}{*}{ Amhara } & Chagni & Spring & 3280 & 4300 \\
\multirow{2}{*}{ Somalia } & Dolloo & Well & 2280 & 3030 \\
& Dolloo (Wardher) & Well & 1950 & 2640 \\
\hline
\end{tabular}




\begin{tabular}{lllll}
\hline Region & Zone, wereda or another village & Source of Sample & Total dissolved solid Concentration $(\mathbf{m g} / \mathbf{L})$ & Electrical conductivity $(\boldsymbol{\mu S} / \mathbf{c m})$ \\
\hline \multirow{6}{*}{ Dolloo (Danod) } & Well & 1540 & 2110 \\
& Dolloo (Danod) & Well & 2000 & 2680 \\
& Dolloo (Danod) & Well & 1730 & 2350 \\
& Addiar Foun & Well & 1660 & 2270 \\
& WakeAdaar & Well & 1560 & 2130 \\
& Mesgidadar Adaar & Well & 3360 & 4430 \\
& Daridaba Adaar & Well & 2330 & 3100 \\
& Talalak (Wata) & Well & 3220 & 4230 \\
& Halbehawaletelalak & Well & 1360 & 1887 \\
\hline
\end{tabular}

Afluoride, total dissolved solid and electrical conductivityin drinking water in the low class indicates samples with no risk for human health. However, water samples higher than the maximum allowable fluoride, total dissolved solid and electrical conductivityadversely affects the public health due to excessive consumption of fluoride, total dissolved solid and electrical conductivity. Generally in the country, $24 \%(n=54), 9.3 \%(n=21)$ and $8.4 \%(n=19)$ of the well water samples, $7.2 \%(\mathrm{n}=7), 1 \%(\mathrm{n}=1)$ and $1 \%$ $(n=1)$ of the piped water samples and $4.6 \%(n=1), 4.6 \%(n=$ 1) and $4.6 \%(n=1)$ of the spring water samples had fluoride, total dissolved solid and electrical conductivity concentration higher than the maximum allowable concentration (i.e. $1.5 \mathrm{mg} / 1,1000 \mathrm{mg} / \mathrm{L}$ and $1500 \mu \mathrm{S} / \mathrm{cm})$ respectively and $42.5 \%$ $(n=96), 35 \%(n=79)$ and $37.2 \%(n=84)$ of the well water samples, $32 \%(\mathrm{n}=31), 0 \%(\mathrm{n}=0)$ and $0 \%(\mathrm{n}=0)$ of the piped water samples and $27.3 \%(n=6), 4.6 \%(n=1)$ and $4.6 \%$ $(n=1)$ of the spring water samples had fluoride, total dissolved solid and electrical conductivity concentration between $0.5 \mathrm{mg} / \mathrm{L}, 500 \mathrm{mg} / \mathrm{l}, 700 \mu \mathrm{S} / \mathrm{cm}$ and the WHO recommendation guideline value (i.e. $1.5 \mathrm{mg} / \mathrm{L}, 1000 \mathrm{mg} / \mathrm{l}$, $1500 \mu \mathrm{S} / \mathrm{cm})$ respectively. In addition to this $33.6 \%(\mathrm{n}=76)$, $55.8 \% \quad(n=126)$ and $54.4 \% \quad(n=123)$ of the well water samples, $60.8 \%(\mathrm{n}=59), 99 \%(\mathrm{n}=96)$ and $99 \%(\mathrm{n}=96)$ of the piped water samples and $68.2 \%(n=15), 91 \%(n=20)$ and $91 \%(\mathrm{n}=20)$ of the spring water samples are below $0.5 \mathrm{mg} / \mathrm{L}$, $500 \mathrm{mg} / \mathrm{l}$ and $700 \mu \mathrm{S} / \mathrm{cm}$ of fluoride, total dissolved solid and electrical conductivity concentration respectively.

In the country, fluoride, total dissolved solid and electrical conductivity content analyzed in the well water sample in the regions had mean value of fluoride, total dissolved solid and electrical conductivity concentration less than the WHO maximum allowable standard of fluoride, total dissolved solid and electrical conductivity $(1.5 \mathrm{mg} / \mathrm{L}, 1000 \mathrm{mg} / \mathrm{L}$ and $1500 \mu \mathrm{S} / \mathrm{cm}$ ) respectively except the average fluoride concentration of Oromia region $(2.47 \mathrm{mg} / \mathrm{L})$ and total dissolved solid and electrical conductivity in the region Somalia $(1246.3 \mathrm{mg} / \mathrm{L}$ and $1723.6 \mu \mathrm{S} / \mathrm{cm})$ and Afar $(1196.1$ $\mathrm{mg} / \mathrm{L}$ and $1647.9 \mu \mathrm{S} / \mathrm{cm}$ ) respectively as indicated in (Table 1, Table 7 and Table 11). Furthermore, as groundwater passes through the earth and comes into contact with fluoride containing minerals, fluoride is dissolved and enters the water. The deeper the water flows through the earth, the more fluoride-containing minerals, and the greater fluoride concentration in the water.

High exposure to Fluoride ion leads to flourosis in its dental and skeletal forms and is endemic in countries, including India, China, Mexico, Argentina, Brazil, Saudi Arabia, United States, Uganda, Tanzania, and Ethiopia. Highrisk areas are mostly located in arid and semi-arid regions that are characterized by a rapid rate of chemical weathering of geological materials, in the center of East African rift, higher levels of salinity and fluoride are the most widely known. Fluoride ion problem is not only clinical, but also social problem too [22]. Ethiopia has surface and ground water resources potential, of which ground water hasalionshare. Several studies conducted in the Rift valley have shown that groundwater contains excess fluoride contents where it adversely affects the health of the surrounding community. Fluoride concentration higher than the WHO guideline value $1.5 \mathrm{mg} / \mathrm{L}$ causes dental fluorosis or mottling of teeth. Few studies of dental fluorosis prior to 1985 in Ethiopia reported high prevalence rates in several communities in the central part of the Rift Valley [32, 33].

The fluoride, total dissolved solid and electrical conductivity content analyzed in the Piped water sample in the regions had mean value of fluoride, total dissolved solid and electrical conductivity concentration less than the WHO maximum allowable standard of fluoride, total dissolved solid and electrical conductivity $(1.5 \mathrm{mg} / \mathrm{L}, 1000 \mathrm{mg} / \mathrm{L}$ and $1500 \mu \mathrm{S} / \mathrm{cm}$ ) respectively (Table 2 , Table 8 and Table 12 ).

The fluoride, total dissolved solid and electrical conductivity content analyzed in the spring water sample in the regions had mean value of fluoride, total dissolved solid and electrical conductivity concentration less than the WHO maximum allowable standard of fluoride, total dissolved solid and electrical conductivity $(1.5 \mathrm{mg} / \mathrm{L}, 1000 \mathrm{mg} / \mathrm{L}$ and $1500 \mu \mathrm{S} / \mathrm{cm}$ ) respectively except the average fluoride concentration of Southern Nation Nationality People Region $(2.62 \mathrm{mg} / \mathrm{L})$ as indicated in (Table 3, Table 9 and Table 13). On the other hand, in the Amhara region of Ethiopia recorded fairly low average content of fluoride, total dissolved solid and electrical conductivity in all the well, piped and spring water samples was below the WHO maximum allowable standard of fluoride $(1.5 \mathrm{mg} / \mathrm{L}, 1000 \mathrm{mg} / \mathrm{L}$ and $1500 \mu \mathrm{S} / \mathrm{cm})$ respectively as indicate in (Table $(1,2,3)$, Table $(7,8,9)$ and Table $(11,12,13))$.

A suitability of ground water for water resource use in the central Ethiopian Rift is hampered by water quality limitations [23]. Over $40 \%$ of deep and shallow wells are contaminated with up to $36 \mathrm{mg} / \mathrm{l}$, sometimes $62 \mathrm{mg} / \mathrm{l}$ of fluoride which is significantly higher than the (WHO) guide line of $1.5 \mathrm{mg} / \mathrm{l}$. [24], that can give rise to a number of adverse effects. Natural ground water quality is mostly 
affected by total dissolved solids, gases and pollutants and is controlled by presence of soluble or reactive minerals in aquifers [25]. As rock chemistry and geological process like weathering of rocks, which promotes the availability of fluoride ions in the ground water [26] needs to be characterized in the study area. Existing methods for defluoridation of drinking water involve expensive and high technology or are slow, inefficient and unhygienic [22, 24]. Also, there is a gap concerning documentation of past experience, evaluating the existing performance of the spatial aquifer system chemical properties and introduction of quality improvement techniques study area. People in several areas of the Ethiopian Rift Valley are consuming water with up to $33 \mathrm{mg} / \mathrm{l}$ of fluoride [34]. So it is required to implement appropriate water treatment procedures using local resources that are accessible to the rural community with technically simple, cost wise feasible and easily transferable technology.

In Amhara region, 7 well water, 1 piped water and 4 spring water samples were analyzed. The average and maximum value of fluoride, total dissolved solid and electrical conductivity concentration detected were $(0.42 \mathrm{mg} / \mathrm{L}, 208.3$ $\mathrm{mg} / \mathrm{L}$ and $308.8 \mu \mathrm{S} / \mathrm{cm})$ and $(1.12 \mathrm{mg} / \mathrm{L}, 433 \mathrm{mg} / \mathrm{L}$ and 636 $\mu \mathrm{S} / \mathrm{cm}) ;(0.34 \mathrm{mg} / \mathrm{L}, 165 \mathrm{mg} / \mathrm{L}$ and $247 \mu \mathrm{S} / \mathrm{cm})$ and $(0.34$ $\mathrm{mg} / \mathrm{L}, 165 \mathrm{mg} / \mathrm{L}$ and $247 \mu \mathrm{S} / \mathrm{cm}) ;(0.66 \mathrm{mg} / \mathrm{L}, 982.5 \mathrm{mg} / \mathrm{L}$ and $1316.8 \mu \mathrm{S} / \mathrm{cm})$ and $(1.09 \mathrm{mg} / \mathrm{L}, 3280 \mathrm{mg} / \mathrm{L}$ and 4300 $\mu \mathrm{S} / \mathrm{cm})$ for well water, piped water and spring water source samples respectively (Table $(1,2,3)$, Table $(7,8,9)$ and Table $(11,12,13)$. Water samples from 1 of those springs (25\%) had total dissolved solid and electrical conductivity concentration above the WHO maximum allowable value of the total dissolved solid and electrical conductivity concentration (Table 10 and 14)).

In Oromia region, 126 well water, 19 piped water and 11 spring water samples were collected and analyzed. The average and maximum value of fluoride, total dissolved solid and electrical conductivity concentration were $(2.47 \mathrm{mg} / \mathrm{L}$, $484.7 \mathrm{mg} / \mathrm{L}$ and $703.5 \mu \mathrm{S} / \mathrm{cm})$ and $(16.96 \mathrm{mg} / \mathrm{L}, 1190 \mathrm{mg} / \mathrm{L}$ and $1666 \mu \mathrm{S} / \mathrm{cm}) ;(1.4 \mathrm{mg} / \mathrm{L}, 305.3 \mathrm{mg} / \mathrm{L}$ and $447.9 \mu \mathrm{S} / \mathrm{cm})$ and $(7.75 \mathrm{mg} / \mathrm{L}, 1170 \mathrm{mg} / \mathrm{L}$ and $1638 \mu \mathrm{S} / \mathrm{cm}) ;(0.23 \mathrm{mg} / \mathrm{L}$, $81.7 \mathrm{mg} / \mathrm{L}$ and $124.1 \mu \mathrm{S} / \mathrm{cm})$ and $(0.58 \mathrm{mg} / \mathrm{L}, 180 \mathrm{mg} / \mathrm{L}$ and $270 \mu \mathrm{S} / \mathrm{cm})$ for well water, piped water and spring water source samples (Table $(1,2,3)$, Table $(7,8,9)$ and Table (11, 12, 13)). Fluoride, total dissolved solid and electrical conductivity concentration above the WHO standard value were detected in $36.5 \%, 1.6 \%$ and $0.8 \%$ of the well water samples $(n=46, n=2$ and $n=1), 26.3 \%, 5.3 \%$ and $5.3 \%$ of the piped water samples $(n=5, n=1$ and $n=1)$, and $0 \%$ of the spring water $(n=0)$ as indicated in (Table 4, 10 and14).

Fluoride, total dissolved solid and electrical conductivity above the WHO standard was detected in some area of the Oromia region (Table 6 and 15). In Lume (Qoqa), Zeway and Adamitulu for fluoride and Sululta for total dissolved solid and electrical conductivity samples from well water, East Shoa(Batu) from piped water had fluoride, total dissolved solid and electrical conductivity concentration which exceeded $(1.5 \mathrm{mg} / \mathrm{L}, 1000 \mathrm{mg} / \mathrm{L}$ and $1500 \mu \mathrm{S} / \mathrm{cm})$. Several cases of fluorosis have been reported from this area. High- risk areas are mostly located in arid and semi-arid regions that are characterized by a rapid rate of chemical weathering of geological materials, in the center of East African rift, higher levels of salinity and fluoride are the most widely known. Fluoride ion problem is not only clinical, but also social problem too [22]. Ethiopia has surface and ground water resources potential, of which ground water hasalionshare. Concentrations of fluoride greater than the WHO guideline value of $1.5 \mathrm{mg} / \mathrm{l}$ have been found in ground waters from several parts of Ethiopia, but are recognized to be highest in the Rift Valley zone [34]. According to [35], in the Ziway-Shala basin in particular, wells had high fluoride levels (mean: $9.4 \pm 10.5 \mathrm{mg} / \mathrm{L}$; range: 1.1 to $68 \mathrm{mg} / \mathrm{L}$ ), with 48 of 50 exceeding the WHO drinking water guideline limit of $1.5 \mathrm{mg} / \mathrm{L}$.

In Addis Ababa, 42 well water and 77 piped water samples were collected from all parts of the city. The average concentration fluoride, total dissolved solid and electrical conductivity in well water, piped water and spring water samples were very low than WHO standard value $(1.5 \mathrm{mg} / \mathrm{L}$, $1000 \mathrm{mg} / \mathrm{L}$ and $1500 \mu \mathrm{S} / \mathrm{cm})$. The mean and maximum value of fluoride, total dissolved solid and electrical conductivity concentration detected were $(0.49 \mathrm{mg} / \mathrm{L}, 301 \mathrm{mg} / \mathrm{L}$ and 444.5 $\mu \mathrm{S} / \mathrm{cm})$ and $(2.81 \mathrm{mg} / \mathrm{L}, 579 \mathrm{mg} / \mathrm{L}$ and $843 \mu \mathrm{S} / \mathrm{cm})$; and $(0.46 \mathrm{mg} / \mathrm{L}, 201.1 \mathrm{mg} / \mathrm{L}$ and $301 \mu \mathrm{S} / \mathrm{cm})$ and $(4.44 \mathrm{mg} / \mathrm{L}, 448$ $\mathrm{mg} / \mathrm{L}$ and $658 \mu \mathrm{S} / \mathrm{cm}$ ) for well water and piped water samples respectively as indicated in the (Table $(1,2,3)$, Table $(7,8,9)$ and Table $(11,12,13))$. Two and two well water samples were analyzed from Harari and Dire Dawa regions respectively. The average and maximum value of fluoride, total dissolved solid and electrical conductivity concentration detected were lower than the WHO maximum allowable standard value as indicated in (Table $(1,2,3)$, Table $(7,8,9)$ and Table $(11,12,13))$.

The Tigray region, 6 well water and 2 spring water samples were analyzed. The average and maximum value of fluoride, total dissolved solid and electrical conductivity concentration detected were $(1.28 \mathrm{mg} / \mathrm{L}, 515.2 \mathrm{mg} / \mathrm{L}$ and $755.3 \mu \mathrm{S} / \mathrm{cm})$ and $(4.18 \mathrm{mg} / \mathrm{L}, 772 \mathrm{mg} / \mathrm{L}$ and $1107 \mu \mathrm{S} / \mathrm{cm})$; and $(0.075 \mathrm{mg} / \mathrm{L}, 207 \mathrm{mg} / \mathrm{L}$ and $309.5 \mu \mathrm{S} / \mathrm{cm})$ and $(0.08$ $\mathrm{mg} / \mathrm{L}, 226 \mathrm{mg} / \mathrm{L}$ and $337 \mu \mathrm{S} / \mathrm{cm}$ ) for well water and spring water samples respectively as depicted at (Table $(1,2,3)$, Table $(7,8,9)$ and Table $(11,12,13))$.. Two from well water $(33.3 \%)$ had fluoride concentration above the maximum allowed levels for drinking water (Table 4). Unacceptable or moderately high concentrations have also been found in ground waters from volcanic rocks in the highlands. Concentrations in ground waters from the ancient basement rocks are typically low for instance in Mekele area [36].

In Somalia region, 16 well water samples were analyzed. The average and maximum value of fluoride, total dissolved solid and electrical conductivity concentration detected were $(1.36 \mathrm{mg} / \mathrm{L}, 1246.3 \mathrm{mg} / \mathrm{L}$ and $1723.6 \mu \mathrm{S} / \mathrm{cm})$ and $(5.1 \mathrm{mg} / \mathrm{L}$, $2280 \mathrm{mg} / \mathrm{L}$ and $3030 \mu \mathrm{S} / \mathrm{cm}$ ) for well water samples respectively as depicted at (Table1, Table7 and Table 11). Fluoride, total dissolved solid and electrical conductivity concentration above the WHO standard value were detected in $12.5 \%, 62.5 \%$ and 
$62.5 \%$ of the well water samples $(n=2, n=10$ and $n=10)$ respectively as indicated in (Table 4, 10 and 14).

The number of well water samples collected and analyzed from different parts of the Afar regions was 20. The average and maximum value of fluoride, total dissolved solid and electrical conductivity concentration detected were $(0.94 \mathrm{mg} / \mathrm{L}, 1196.1 \mathrm{mg} / \mathrm{L}$ and $1647.9 \mu \mathrm{S} / \mathrm{cm})$ and $(2.54 \mathrm{mg} / \mathrm{L}$, $3360 \mathrm{mg} / \mathrm{L}$ and $4430 \mu \mathrm{S} / \mathrm{cm})$ for well water samples respectively in the (Table 1, Table 7 and Table 11). Fluoride, total dissolved solid and electrical conductivity concentration above the WHO standard value were detected in $10 \%, 45 \%$ and $40 \%$ of the well water samples $(n=2, n=9$ and $n=8)$ respectively as indicated in (Table 4, 10 and 14). In Dulesa for fluoride and Addiar foun, Wake Adaar, Mesgidadar Adaar, Daridaba Adaar, Talalak (Wata), Halbehawaletelalak and Yorengira Telalak for total dissolved solid and electrical conductivity samples from well water had fluoride, total dissolved solid and electrical conductivity concentration which exceeded $(1.5 \mathrm{mg} / \mathrm{L}, 1000 \mathrm{mg} / \mathrm{L}$ and $1500 \mu \mathrm{S} / \mathrm{cm})$ in (Table 6 and 15) respectively. The problematic fluoride concentrations were derived from hot springs (high temperature) and by weathering of the volcanic bedrocks [37]. Water sources with Fluoride content above $5.0 \mathrm{mg} / \mathrm{L}$ in the Rift Valley were found mostly in hot spring (100\% of all sources), lakes (78\%), shallow wells (54\%) and boreholes (35\%) and the lowest concentrations less than $1.5 \mathrm{mg} / \mathrm{L}$ observed in springs and rivers [38].

The Southern Nation Nationality People Region is another region where the fluoride, total dissolved solid and electrical conductivity problem is prevalent. 5 well water and 5 spring water samples were collected and analyzed. In this region, drinking water sources with fluoride contents exceeding 1.5 $\mathrm{mg} / \mathrm{L}$ were recorded in $20 \%(\mathrm{n}=1)$ and $20 \%(\mathrm{n}=1)$ for well water and spring water samples respectively as showed in Table 4. Both dental and skeletal fluorosis is prevalent in the Rift Valley region of Ethiopia because of high fluoride waters that originate from springs and boreholes.

\section{Conclusion}

The result of this retrospective study indicated that fluoride, total dissolved solid and electrical conductivity concentration in the water sample varied from $0.0 \mathrm{mg} / \mathrm{Lto}$ $16.96 \mathrm{mg} / \mathrm{L}, 0.25 \mathrm{mg} / \mathrm{L}$ to $3360 \mathrm{mg} / \mathrm{L}$ and $2.04 \mu \mathrm{S} / \mathrm{cm}$ to $4430 \mu \mathrm{S} / \mathrm{cm}$ respectively. Generally in water samples that was brought to the environmental health laboratory of EPHI in the year between April 2017 to December 2018, $24 \%(\mathrm{n}=54), 9.3 \%(\mathrm{n}=21)$ and $8.4 \%(\mathrm{n}=19)$ of the well water samples, $7.2 \%(n=7), 1 \%(n=1)$ and $1 \%(n=1)$ of the piped water samples and $4.6 \%(n=1), 4.6 \%(n=1)$ and $4.6 \%(n=1)$ of the spring water samples had fluoride, total dissolved solid and electrical conductivity concentration higher than the maximum allowable concentration (i.e. $1.5 \mathrm{mg} / 1,1000 \mathrm{mg} / \mathrm{L}$ and $1500 \mu \mathrm{S} / \mathrm{cm}$ ) respectively and $42.5 \%(\mathrm{n}=96), 35 \%(\mathrm{n}=79)$ and $37.2 \%(n=84)$ of the well water samples, $32 \%(n=31)$, $0 \%(n=0)$ and $0 \%(n=0)$ of the piped water samples and
$27.3 \%(\mathrm{n}=6), 4.6 \%(\mathrm{n}=1)$ and $4.6 \%(\mathrm{n}=1)$ of the spring water samples had fluoride, total dissolved solid and electrical conductivity concentration between $0.5 \mathrm{mg} / \mathrm{L}$, $500 \mathrm{mg} / 1,700 \mu \mathrm{S} / \mathrm{cm}$ and the WHO recommendation guideline value (i.e. $1.5 \mathrm{mg} / \mathrm{L}, 1000 \mathrm{mg} / 1,1500 \mu \mathrm{S} / \mathrm{cm}$ ) respectively. In addition to this $33.6 \% \quad(\mathrm{n}=76)$, $55.8 \%(n=126)$ and $54.4 \%(n=123)$ of the well water samples, 60.8\% $(n=59), 99 \%(n=96)$ and $99 \%(n=96)$ of the piped water samples and $68.2 \%(n=15), 91 \%(n=20)$ and $91 \%(n=20)$ of the spring water samples are below 0.5 $\mathrm{mg} / \mathrm{L}, 500 \mathrm{mg} / 1$ and $700 \mu \mathrm{S} / \mathrm{cm}$ of fluoride, total dissolved solid and electrical conductivity concentration respectively. A concentration of fluoride, total dissolved solid and electrical conductivity below $0.5,500$ and 700 were recorded in $63 \%, 97.5 \%$ and $97.5 \% ; 75 \%, 50 \%$ and $50 \%$; and $58.3 \%, 91.7 \%$ and $91.7 \%$ of samples collected from Addis Ababa, Tigray and Amhara regional states respectively. Therefore, to decide the importance of fluoride and fluoridation in the prevention of Dental Caries or Tooth decay for water sources with fluoride concentration of $0.0 \mathrm{mg} / \mathrm{L}$ or below $0.5 \mathrm{mg} / \mathrm{L}$ is needed more research. However, water fluoridation is still opposed by some scholars; reasons for opposition include concerns about possible long-term harmful effects such as a high risk of Osteoporosis. Rift Valley areas of Afar, SNNPR and Oromia are characterized with a highest concentration of fluoride. The concentration below $1.5 \mathrm{mg} / \mathrm{L}$ carry an increasing risk of dental fluorosis, and higher concentrations lead to skeletal fluorosis especially to the Rift Valley area. Therefore, the result of this retrospective study will use as a base to health authorities as well as other responsible body for the management of water supply regarding fluoride.

\section{References}

[1] Patil, S. B., Veena, Dr., Soraganvi, S., 2017. Geochemical Analysis for Fluoridein Ground waterof Malaprabha River Basin Using GIS. International Journal of Engineering Sciences Research Technology ISSN: 2277-9655. http://www.ijesrt.com/.

[2] Rango, T., et al., 2012. Ground water quality and its health impact: An assessment of dental flourosis in rural inhabitants of the Main Ethiopian Rift.Environ.Int.143 (2012), 37-47 Duke University, U.S

[3] Bruvold WH and Ongerth HJ (1969). Taste quality of mineralized water. Journal of the American Water Works Association, 61: 170.

[4] Moujabber M E, Samra B B, Darwish T and Atallah T 2006 Comparison of different indicators for groundwater contamination by seawater intrusion on the Lebanese coast Water Resour. Manag. 20 161-180.

[5] Stigter T Y, Ribeiro L and Carvalho D 2006 Application of a groundwater quality index as an assessment and communication tool in agro-environmental policies - Two Portuguese case studies J. Hydrol 327 578-891. 
[6] Nonner J C 2015 Introduction to Hydrogeology (London: CRC Press, Taylor and Francis Group).

[7] Han D, Kohfahl C, Song X, Xiao G and Yang J 2011 Geochemical and isotopic evidence for palaeo-seawater intrusion into the south coast aquifer of Laizhou Bay, China, Appl. Geochemistry 26 863-883.

[8] Patil P N, Sawant D V, and Deshmukh R N, 2012 Physicochemical parameters for testing of water - a review, Int. J. Environ. Sci. 3 1194-1207.

[9] Marandi A, Polikarpus M and Jõeleht A 2013 A new approach for describing the relationship between electrical conductivity and major anion concentration in natural waters Appl. Geochemistry 38 103-109.

[10] Daniels W L, Zipper C E, Orndorff Z W, Skousen J, Barton C D, McDonald L M and Beck M A 2016 Predicting total dissolved solids release from central Appalachian coal mine spoils Environ. Pollut. 216 371-379.

[11] Kumar S K, Logeshkumaran A, Magesh N S, Godson P S and Chandrasekar N 2015 Hydrogeochemistry and application of water quality index (WQI) for groundwater quality assessment, Anna Nagar, part of Chennai City, Tamil Nadu, India Appl. Water Sci. 5 335-343.

[12] Hem D 1985 Study and Interpretation the Chemical of Natural of Characteristics Natural Water 3rd edition USGS Water Supply Paper 2254 66-69 US Govt Printing Office Washington DC.

[13] Appelo C A J and Postma D 2005 Geochemistry, groundwater and pollution (Amsterdam: CRC Oress, Taylor \& Francais Group).

[14] Carreira P M, Marques J M and Nunes D 2014 Source of groundwater salinity in coastline aquifers based on environmental isotopes (Portugal): Natural vs. human interference. A review and reinterpretation Appl. Geochemistry 41 163-175.

[15] Rusydi A F, Naily W and Lestiana H 2015 Pencemaran Limbah Domestik Dan Pertanian Terhadap Airtanah Bebas Di Kabupaten Bandung J. Ris. Geol. dan Pertamb. 25 87-97.

[16] WHO 2011 WHO guidelines for drinking-water quality (Geneva: World Health Organization).

[17] Todd D K and Mays L W 2005 Groundwater Hydrology ed B Zobrist (New Jersey: John Wiley \& Sons, Inc.).

[18] Rhoades J, Kandiah A and Mashali A 1992 the use of saline waters for crop production (Rome: FAO United Nations).

[19] TorA. Removal of fluoridefroman aqueous solution by using montmorillonite. Desalination 2006; 201 (1-3): 267-76.

[20] Isogai A, Nakagaki H, Hanaki M, Tsuboi S, Morita I, Osaka C. Use of fluoridated dentifrice and glucoseretention at the approximal areas of anterior teeth. ASDCJ DentChild2001; 68 (1): 42-6, 12.

[21] Jones S, Burt BA, Petersen PE, LennonMA. The effective use of fluoride in public health. BullWorldHealthOrgan2005; 83 (9): 670-6.

[22] Yadav, Rajdeep, Yadav, R. N., Chandrawa, M. P. S., Sharma, Sanjay K., 2008. Assessment of Fluoride Content, Ph and TDS in.

[23] Kebede, Wolka, Mengistu, Tefera, Taddese, Habitamu, Tolera,
Alemayehu, 2014. Impact of land cover change on water quality and stream flow in lake Hawassa watershed of Ethiopia. Wondo Genet College of Forestry and Natural Resources, Hawassa University, Hawassa, Ethiopia. Agricultural Sciences 5, 647-659. https://www.scirp.org/journal/PaperInformation.aspx?PaperID=47 641.

[24] Rango, T., et al., 2014. Mobilization of Arsenic and Other Naturally Occurring Contaminants in Ground water of the Main Ethiopian Rift Aquifers. Division of Earth and Ocean Sciences, Nicholas School of the Environment. Duke University, Durham, United States.

[25] FDRE Ministry of Water Resources, Ethiopian Water Technology Centre, 2008. Butajira Ziway Areas Development Study of Water Quality. Addis Ababa, Ethiopia

[26] Rafique, T., Naseem, S., Bhanger, Mohamed. I., Tanzil, Bhanger, Usmani, H., 2008. Fluoride ion contamination in the ground water of Mithisubdistrict, the Thar Desert, Pakistan. Springer. Environmental Geology. Int. J. Geosci.56 (2), 317326. Online ISSN 14320495 https://link.springer.com/article/10.1007/s00254007-1167-y.

[27] Haimanot, Reda Tekle, Fekadu, A. and Bushra, B. (1987), "Endemic fluorosis in the Ethiopian Rift Valley", Tropical and Geographical Medicine, vol. 39, No. 3, pp. 209-217.

[28] FDRE, (1995) Availableat: http://www.ethiopia.gov.et/regionalstates (accessed 13 december 2018).

[29] APHA, (1992), Standared Methods for the Examination of water and wastewater. Edited by Greenberg, Arnold E. Clesceri, Lenore S. and Eaton, Andrew D., American Public Health Association, Washington, DC 20005.

[30] WHO, (1971), "WHO guidelines for fluoride in drinking water".

[31] Guidelines on health aspects of water desalination. ETS/80.4. Geneva: World Health Organization, 1980.

[32] Abdo, A. (1978), "A Survey of Dental Fluorosis in Abossa Elementary School”, Ethiopian Medical Journal, vol. 16, pp. 138.

[33] Olsson, B. (1979), "Dental findings in high-fluoride areas in Ethiopia", Community Dental and Oral Epidemiology, vol. 7, pp. 51-56.

[34] Kloos, Helmut and Haimanot, Reda Tekle (1993), "Fluorosis in the ecology of health and disease in Ethiopia".

[35] Rango, T., Kravchenko, J., Atlaw, B., McCornick, Peter G., Jeuland, M., Merola, B. and Vengosh, A. (2012), "Groundwater quality and its health impact", An assessment of dental fluorosis in rural inhabitants of the Main Ethiopian Rift, ELSEVIER, vol. 43, pp. 37-47.

[36] Chernet, T. and Eshete, G. (1982), "Hydrogeology of the Mekele area", Ethiopian Institute of Geological survey, Addis Ababa, Memoire no. 2.

[37] Ashley, R. P. and Burley, M. J. (1994), "Controls on the occurrence of fluoride in ground water in the rift valley of Ethiopia”.

[38] Kloos, Helmut and Haimanot, Reda Tekle (1999), "Distribution of fluoride and fluorosis in Ethiopia and Prospective for control", Tropical Medicine and International Health, vol. 4, No. 5, pp. 355-364. 